https://doi.org/10.15407/ujpe65.6.548

\title{
ACADEMICIAN \\ OF THE NAS OF UKRAINE \\ VADYM MYKHAILOVYCH LOKTEV \\ (to the 75th anniversary of his birthday)
}

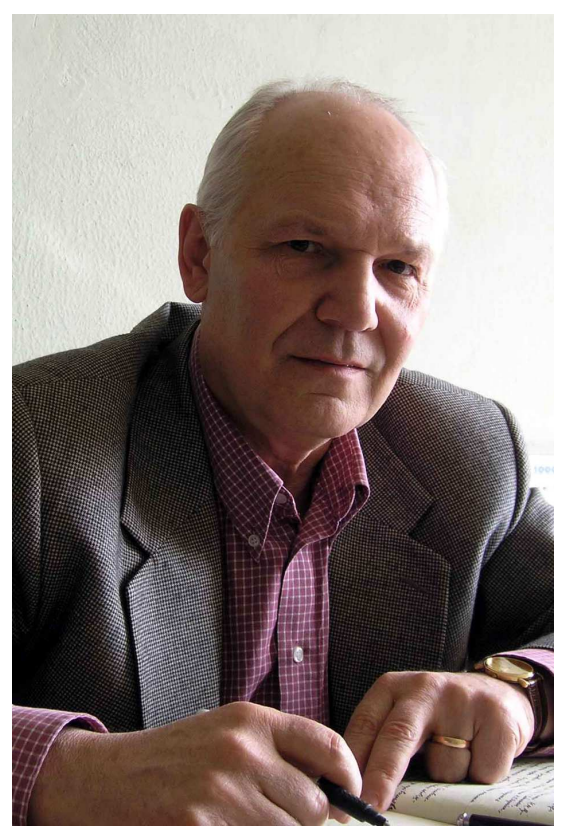

May 3, 2020 marked the 75th birthday of the outstanding physicist-theorist, Doctor of Science in Physics and Mathematics, Professor, Honored Worker in Science and Engineering of Ukraine, Laureate of two State Prizes of Ukraine, Academician of the National Academy of Sciences of Ukraine (NASU), a member of the Presidium of the NASU, AcademicianSecretary of the Department of Physics and Astronomy of the NASU Vadym Mykhailovych Loktev.

Vadym Loktev was born on May 3, 1945 in Kyiv. After finishing the secondary school No. 100, he entered the Faculty of Radiophysics of Taras Shevchenko State University in Kyiv. Later, when being a 3rd-year student, he had realized that he wanted to major in theoretical physics. Therefore, he transferred to the Faculty of Physics of the same University, from which he successfully graduated in 1968. The same year he became a postgraduate student at the M.M. Bogolyubov Institute for Theoretical Physics (BITP) of the NASU with which he linked all his further life. He started to work there in 1967 under the supervision of Academician of the NASU O.S. Davydov.

At that time, the significant progress was achieved in the experimental study of the spectra of solid oxygen that was carried out at the Institute of Physics of the NASU under the leadership of Academician of the NASU Antonina Fedorivna Prykhotko. The results obtained required a theoretical explanation. Solid oxygen is a unique crystal in which the properties of a molecular crystal and a magnet are combined. Despite that solid oxygen was experimentally obtained as long ago as at the beginning of the twentieth century, its study has been continued till now. In particular, the important research of the optical and magnetic properties of solid oxygen and antiferromagnetic insulators were performed in a group of theorists headed by E.G. Petrov at the BITP. O.S. Davydov included his young talented student, Vadym Loktev, into this group. The work progressed rapidly, and in 1971, under the supervision of O.S. Davydov and E.G. Petrov, Vadym Loktev defended his Ph.D. dissertation. Later, in 1983, V.M. Loktev defended his doctoral dissertation.

It was a period, when the main features of Vadym Loktev as a scientist were created. He is one of the most prominent representatives of the world-famous Davydov's scientific school. He made significant efforts to continue the traditions and develop the ideas

ISSN 2071-0194. Ukr. J. Phys. 2020. Vol. 65, No. 6 
of this school, to engage new persons into it, and to expand the field of its scientific activity, always remaining at the forefront of science. In 1997, Vadym Loktev received the title of Professor. The same year, V.M. Loktev was elected Corresponding Member and, in 2003, Academician of the NASU.

V. Loktev's managerial abilities manifested themselves from the very beginning of his scientific career. In particular, in 1987, he was elected the Head of the Laboratory of electronic processes in ordered molecular structures of the Department of the Theory of Many-Particle Systems. The latter was headed by Academician of the NASU O.S. Davydov. From 1993 to 2016, V.M. Loktev headed the Department of Nonlinear Condensed Matter Physics. Since 2004 he is Academician-Secretary of the Department of Physics and Astronomy (DPA) of the NASU. At this position, he initiated and made significant efforts to organize and successfully hold scientific meetings of the DPA of the NASU by engaging both well-known experts and young researchers into this activity.

The scientific achievements of Vadym Loktev include significant results obtained in many branches of modern science. These are the theories of cryocrystals, magnetic phenomena, disordered systems, superconductivity (in particular, high-temperature superconductivity), and spin-orbit interaction. It is impossible to cover all important results obtained by Vadym Loktev in a brief communication. Even their list would take more than a page. Therefore, let us recall only the most notable of them.

As a result of the close cooperation with Academicians of the NASU O.S. Davydov and A.F. Prykhotko, as well as together with his colleague and close friend Yurii Gaididei, Vadym Loktev explained a number of experimental data obtained for cryocrystals, in particular, for solid oxygen. He obtained some fundamental results in the theory of molecular and magnetic excitons and phase transitions in condensed systems.

Since the early years of his scientific activity, such a close cooperation with experimenters stimulated Vadym Loktev not to stop at the theoretical results obtained in the analytical form, but also to analyze their possible experimental consequences and make some important scientific predictions. First of all, this is the splitting of bi-exciton absorption bands in antiferromagnetic insulators, which was predicted together with Yu.B. Gaididei, the polarization of spec- tral lines in the region of two-particle light absorption, and the prediction of a magnetic structure in one of the low-temperature phases of solid oxygen (it was called the Loktev structure). It is also a prediction (made together with V.S. Ostrovskyi) of a new magneto-optical effect, linear in the external magnetic field, in anisotropic magnetic crystals, which was previously considered impossible. On the basis of this prediction, the experimenters managed to visualize collinear domains in antiferromagnets. V.M. Loktev developed the theory of the Rashba effect in antiferromagnets and determined the possibility of collective rearrangement in their spectra irrespective of the impurity concentration. He proposed an explanation for the anomalous enhancement of the infrared absorption in disordered antiferromagnets and developed the corresponding theory, which became known as the Ivanov-Loktev-Pogorelov theory. Vadym Loktev created a theory of spin excitations in disordered magnets with strong single-ion anisotropy and a theory describing spin waves and the formation of a magnetoelastic gap in quasi-two-dimensional antiferromagnets. He together with V.M. Kalita developed the concept of quantum magnetic phase transitions of the displacement type.

V.M. Loktev proposed a quantum theory of the linear and nonlinear magnetic properties of magnets with a strong contribution of the spin-orbit interaction, for which the capabilities of the LandauLifshitz theory are limited. He discovered a new type of magnetoelastic excitations (magneto-bending waves) and developed their theory (together with V.G. Bar'yakhtar and S.M. Ryabchenko).

Since the very beginning of the discovery of high-temperature superconductivity in cuprate compounds, when it became clear that this phenomenon has a number of features that distinguish it from superconductivity in earlier known systems (the latter can be described with high accuracy in the framework of the Bardeen-Cooper-Schrieffer theory), Vadym Loktev actively participated the development of the theory of this phenomenon. Together with V.P. Gusynin and S.G. Sharapov, he generalized the Bardeen-Cooper-Schrieffer theory to the case of metal systems with an arbitrary charge carrier concentration, which made it possible to describe the crossover from superfluidity to superconductivity and the appearance of a pseudogap in the quasiparticle spectrum of charge carriers. V.M. Loktev ana- 
lyzed the properties of high-temperature superconducting compounds as narrow-band conductors, the initial phases of which belong to typical antiferromagnetic insulators, and the metallic ones to the socalled bad metals. Together with Yu.G. Pogorelov, he developed a theory of superconductivity in doped, or the so-called bad, metals, in which the formation of a superconducting condensate is only possible within a finite interval of the charge carrier concentration (this phenomenon does take place in hightemperature superconducting compounds). Together with Yu.B. Gaididei, he substantiated a non-phonon pairing mechanism in high-temperature superconductors, the so-called Gaididei-Loktev-Weber mechanism. Together with V.G. Bar'yakhtar, he proposed a full phenomenological theory of static and resonance properties of nonconducting phases of hightemperature superconductors and determined their magnetic structures. The values of the critical fields calculated by him for the magnetic phase transformations, as well as the calculated spin excitation spectra, were confirmed experimentally with high accuracy.

V.M. Loktev obtained a number of significant results, while studying impurity states in quasi-twodimensional materials. For example, a possibility for impurity levels to be formed in graphene was shown together with Yu.V. Skrypnyk. They considered peculiarities in the band structure of those systems in vicinities of the singular points (like Dirac points for single-layer graphene or nodal points for hightemperature cuprates) and singular curves (inflection curves for two-layer graphene) in their energy spectrum. The parameters of newly formed impurity bands or gaps were determined. Conditions for the quasiparticle localization in those spectral regions were analyzed, and the corresponding mobility limits were found. For quasiparticles near the Dirac points in single-layer graphene, the preservation of their coherent states in the presence of substitution impurities was proved. It was also shown that their states become destroyed, if an arbitrarily small amount of vacancies is available. This circumstance opens an opportunity to induce, or to stimulate, metal-insulator phase transitions with the help of a certain external modulation of the Fermi level by varying the mobility limit.

For two-layer graphene with a gap in the spectrum, V.M. Loktev found that, under the action of an external electric field, the impurities can provoke an even more complicated reconstruction of the spectrum giving rise to the formation of a narrow impurity band in the gap and holding the mobility limit within this band. As a result, a number of quantitative changes and electronic phase transitions can take place under the action of a rather weak additional external field. By an example of doped silicene, it was shown that, because of the peculiarities in its honeycomb structure, analogous transformations in its spectrum can occur even in a one-layer system provided that the latter is sufficiently ribbed. Therefore, even a weak external modulation can become responsible for a substantial change in the electrical resistance and other observed properties (optical, thermal, and so forth) of silicene. For those results, Vadym Loktev (together with Yurii Pogorelov) was awarded the Davydov Prize of the BITP of the NASU in 2019.

Vadym Loktev was the first who established the important role of the Jahn-Teller effect in the mechanism of high-temperature superconductivity in fullerite films (together with E.A. Pashitskii). Together with Yu.M. Khalak, he also predicted a possibility of the formation of an orientational domain structure in fullerite.

The formation of domains in antiferromagnets associated with the magnetoelastic interaction, the destressing phenomenon, and the shape effect (the dependence of the frequencies of antiferromagnets on the shape of their specimens) were also predicted by V.M. Loktev. Together with H.V. Gomonay, a theory of torque induced by a spin-polarized current in antiferromagnets was developed, the origins of the threshold excitation of acousto-luminescence were found, and the character of the relief for the step motion at helical dislocations was elucidated. Together with A.I. Bugrii, V.M. Loktev created a theory of space-inhomogeneous Bose-Einstein condensation of magnons in thin ferromagnetic films, which can take place at high temperatures, including room temperature.

The study of nonmagnetic systems with helical symmetry carried out by V.M. Loktev together with O.O. Yeremko showed that they can possess spintronic properties.

Vadym Loktev studied the influence of an external field on the spin states of electrons and obtained, in the nonrelativistic approximation, a generalized operator that describes this influence in the Schrödinger equation, the so-called spin-orbit interaction opera-

ISSN 2071-0194. Ukr. J. Phys. 2020. Vol. 65, No. 6 
tor. Together with L.S. Brizhik and O.O. Yeremko, he showed that this operator includes not only the well-known Thomas-Frenkel correction term but also a new, additional contribution, which was unknown earlier and which was called the Brizhik-YeremkoLoktev operator.

The brief summary of the results obtained by Vadym Mykhailovych, which was presented above, testifies to his profound knowledge of physics, extensive erudition, interest in new phenomena, and a capability to study them thoroughly. The body of scientific publications authored and co-authored by V.M. Loktev includes more than 300 scientific papers, as well as several monographs and textbooks. He visited scientific institutions in many countries around the world. He also participated as a guest lecturer at numerous international conferences and symposia. Furthermore, he was an organizer of many of them.

The merits of Vadym Loktev in the pedagogical activity are also substantial. In particular, since 1982 and for 15 years, he is lecturing at the Faculties of Physics and Radiophysics of Taras Shevchenko National University of Kyiv. Since 1998, he has been the Head (part-time) of the Department of General and Theoretical Physics at the National Technical University of Ukraine "Igor Sikorsky Kyiv Polytechnic Institute". Under his supervision, more than two dozen Ph.D. dissertations and a doctoral one were defended. He actively participates in the physics education of young people not only at the universities, but starting from school years. For this purpose and with his active participation, a textbook on physics for high school classes had been written, because, from his own experience as a lecturer, he saw how low the level of knowledge of first-year students in physics felt down.

As a scientific manager, Academician V.M. Loktev is a member of the International Advisory Board of the scientific journal Fizika Nizkikh Temperatur (Low Temperature Physics), a member of the editorial boards of the journals Dopovidi NAN Ukrainy
(Reports of the NAS of Ukraine), Ukrainskyi Fizychnyi Zhurnal (Ukrainian Journal of Physics), Visnyk NAN Ukrainy (Bulletin of the NAS of Ukraine), and Condensed Matter Physics. He is a chair of the section "Solid State Theory" of the Scientific Council on the trend "Physics of the Metallic State", and the Deputy Chair of the Scientific Council on Physics of the Ministry of Education and Science of Ukraine. In 1997-2004, Academician V.M. Loktev was the chair of the Interdepartmental Scientific Council "Hightemperature superconductivity"; in 1997-2003, the organizer and permanent chair of the scientific sessions of the DPA of the NASU; and in 2004, he was elected Academician-Secretary of the DPA and a member of the Presidium of the NASU. Since 2013, he has been the Editor-in-Chief of the Great Ukrainian Encyclopedia.

The social-political activity of Vadym Loktev cannot be ignored. Being concerned about the state of science in Ukraine and understanding its crucial role in the development of Ukraine as an independent state, he wrote many articles in Ukrainian journals, appeared on television, and gave numerous interviews.

V.M. Loktev was awarded the Orders of Prince Yaroslav the Wise of the 5th, 4th, and 3rd classes, an Honorary Diploma of the Supreme Council of Ukraine, the title of Honored Worker in Science and Engineering of Ukraine, and V.I. Vernadskyi Gold Medal. He is a laureate of two State Prizes of Ukraine, K.D. Sinelnikov and M.M. Bogolyubov Prizes of the NASU, and O.S. Davydov Prize of the BITP of the NASU. In 2001, V.M. Loktev was elected a full member of the European Academy (Paris).

Vadym Mykhailovych, we sincerely congratulate you on your birthday! We wish you good health, high inspiration, new creative successes, and fulfillment of your dreams!

A.G. ZAGORODNY, YU.I. IZOTOV, E.G. PETROV, B.I. LEV, V.P. GUSYNIN, YU.A. SITENKO, L.S. BRIZHIK 